\title{
Telomere Protective Effects of a Cyanobacteria Phycocyanin against Blue Light and UV Irradiations: A Skin Anti-Aging and Photo-Protective Agent
}

\author{
Francine Joly ${ }^{1}$, Jean-Eric Branka ${ }^{2}$, Eric Darnis ${ }^{3}$, Luc Lefeuvre ${ }^{4}$ \\ ${ }^{1}$ Sephra, 87 rue Voltaire, Puteaux, France \\ ${ }^{2}$ Ephyscience, 21 rue La Noue Bras de Fer, Nantes, France \\ ${ }^{3}$ Gynecology-Obstetrics, Hospital "Mère et Enfant", Nantes, France \\ ${ }^{4}$ Laboratoires Dermatologiques d'Uriage, Neuilly-sur-Seine, France \\ Email: jebranka@ephyscience.fr
}

How to cite this paper: Joly, F., Branka, J.-E., Darnis, E. and Lefeuvre, L. (2019) Telomere Protective Effects of a Cyanobacteria Phycocyanin against Blue Light and UV Irradiations: A Skin Anti-Aging and Photo-Protective Agent. Journal of Cosmetics, Dermatological Sciences and Applications, 9, 336-345.

https://doi.org/10.4236/jcdsa.2019.94031

Received: October 17, 2019

Accepted: December 9, 2019

Published: December 12, 2019

Copyright $\odot 2019$ by author(s) and Scientific Research Publishing Inc. This work is licensed under the Creative Commons Attribution International License (CC BY 4.0).

http://creativecommons.org/licenses/by/4.0/

\section{cc) (i) Open Access}

\begin{abstract}
Background: Cyanobacteria phycocyanins (Cps) have already shown powerful antioxidant properties. In human cells submitted to oxidative stress the telomeres length decrease, the expression of progerin and the activity of mTOR are increased. At our knowledge, there is no published data on $C p s$ correlated with ultraviolet radiation (UV) and blue light effects in human cells regarding telomeres' length, progerin expression or mTOR1 complex activity. Objectives: In this study, we sought to assess 1) telomeres' length in newborn human fibroblasts exposed to UV and blue light; 2) progerin production in mature human normal fibroblasts exposed to UV; 3) mTOR1 activation in adult human normal keratinocytes exposed to UV, analyzing the activity of a Cyanobacteria phycocyanin $(C P)$ in these in vitro models. Materials and Methods: Human skin fibroblasts or human normal keratinocytes were cultured-in the absence or in the presence of $C P$ and submitted to UVB + UVA and blue light irradiations. Telomeres' length, progerin expression and mTOR1 activity were then assessed by molecular biology and immuno-enzymatic methods. Results: In cultured fibroblasts exposed to irradiations and treated by $\mathrm{Cp}$, telomeres' shortage and progerin expression were lower compared to irradiated untreated cells. In cultured keratinocytes treated by $\mathrm{Cp}$ and exposed to irradiations, the mTOR activity was lower compared to irradiated untreated cells. Conclusions: In these in vitro studies on human skin fibroblasts and on normal human keratinocytes, the cyanobacteria phycocyanin ( $C P$ ) showed a decrease of damages induced by UV and
\end{abstract}


blue light expressed by telomeres preservation and downregulation of progerin expression and of mTOR activity, thus showing skin anti-aging and photo-protective potential.

\section{Keywords}

Cyanobacteria Phycocyanins, UVA, UVB, Blue Light, Telomere Length, Telomere Shortage, Progerin Production, mTOR1 Activity, Human Skin Cells, Aging/Photo-Aging

\section{Introduction}

Cyanobacteria phycocyanins are phycobiliproteins which have been described as potential bioactive compounds and recognized as high-valued natural products for biotechnological applications. They were notably associated to antioxidant, anticancer and anti-inflammatory capacities (for a review, see [1]). As oxidative stress has been associated for a long time with skin aging (for a review, see [2] [3] [4]), phycocyanins have been naturally used in cosmetic products in order to reduce the impact of time on the skin appearance; but they were also used in dermocosmetic to improve wound healing [5] and for their antimelanogenic effect [6]. However, relatively little is known about the cellular signaling pathways they modulate to produce these effects.

As recent researches showed that progerin production and telomere dysfunction collaborate to trigger cellular senescence in normal human skin cells [7], we decided to study the effect of our cyanobacteria phycocyanins on these two parameters by quantifying its action on telomere length and progerin production in models of human normal dermic fibroblasts. Additionally, as UV and blue light irradiations are known to induce oxidative damages and pro-aging effects in skin cells (for a review, see [8]), we also decided to study the effect of these irradiations on human fibroblast progerin production and telomere length and subsequently, to evaluate the effect of our phycocyanin on the possible modifications of these parameters by "UVA/UVB/blue light" irradiations.

To go further in our investigations, we led studies based on the work of Cao et al. [9] which report that rapamycin suppresses a pro-senescent phenotype in progeric cells not only suggesting a non-toxic therapy for progeria but also implying its similarity with normal aging. In fact, rapamycin can decrease intracellular levels of progerin which is responsible for nuclear abnormalities, mitotic abnormalities and accelerated telomere shortening; DNA damage response, p53 induction and cell cycle arrest are then suppressed [10] [11] [12] [13] [14]. In their work, Cao et al. spoke about 4 different scenarios to link progeria to aging: One involves an increased progerin production and telomere shortening in aged cells, and the 3 others emphasize the role of mTOR1 complex, the target of rapamycin, which is extensively involved in cellular biology including autophagy [15], macromolecule biosynthesis [16], cell cycle [17], growth [18], and meta- 
bolism [19]. Obviously, we then chosen to study the effect of our cyanobacteria phycocyanin on the activation rate of mTOR1 complex in a model of human normal keratinocytes submitted to UV radiations.

\section{Materials and Methods}

\subsection{Cell Culture and Treatments}

For telomere length assay

Human normal neonatal fibroblasts were seeded into petri dishes at a cell density of 500,000 cells per petri dish $\left(9090 \mathrm{cells} / \mathrm{cm}^{2}\right)$ in DMEM medium and were cultured at $37^{\circ} \mathrm{C}$ in an atmosphere containing $5 \%$ of $\mathrm{CO}_{2}$.

After reaching confluency, cells were incubated in the absence (control) or in the presence of a cyanobacteria phycocyanin at $0.2 \%$ for 24 hours in medium without supplements at $37^{\circ} \mathrm{C} / 5 \% \mathrm{CO}_{2}$. After this incubation period, cells were irradiated or not (control) with UVA $4 \mathrm{~J} / \mathrm{cm}^{2}+\mathrm{UVB} 0.075 \mathrm{~J} / \mathrm{cm}^{2}$ and Blue light $20 \mathrm{~J} / \mathrm{cm}^{2}$ in $\mathrm{PBS}$ and then incubated at $37^{\circ} \mathrm{C} / 5 \% \mathrm{CO}_{2}$ for a new period of 24 hours.

At the end of this new incubation period, telomeres length was assessed by using a high-throughput (HT) Q-FISH technique.

Each experimental condition was realized in triplicate $(\mathrm{n}=3)$.

For progerin assay

Human normal fibroblasts were seeded into 24-well plates at a cell density of 20,000 cells per well in Dulbecco Modified Eagle Medium (DMEM, Dominique Dutscher, Illkirch, France)/Ham's F12 (Pan Biotech, Aidenbach, Germany) (50:50) containing $10 \%$ of fetal bovine serum (Dominique Dutscher, Illkirch, France) and were cultured at $37^{\circ} \mathrm{C}$ in an atmosphere containing $5 \%$ of $\mathrm{CO}_{2}$ until reaching confluency.

After reaching confluency, cells were cultured in DMEM/Ham's F12 containing $2 \%$ of fetal bovine serum and were pre-incubated in the absence (control) or in the presence of increasing concentrations of a cyanobacteria phycocyanin $(0.02 \% ; 0.2 \%$ and $2 \%, \mathrm{v} / \mathrm{v})$ for 1 hour. At the end of the pre-incubation period, cells were irradiated or not by UVA at $4 \mathrm{~J} / \mathrm{cm}^{2}$ and UVB at $75 \mathrm{~mJ} / \mathrm{cm}^{2}$. At the end of the irradiation, cells were then incubated for 96 hours in the same experimental conditions.

At the end of the incubation period, progerin was extracted from cell monolayer by sonication and then quantified by ELISA.

Total proteins contained in the cell lysates were also quantified, by using a spectro-colorimetric method (Bradford method [20]).

Each experimental condition was realized in triplicate $(\mathrm{n}=3)$.

For mTOR activation assay

Human normal keratinocytes were seeded into 24-well plates at a cell density of 30,000 cells per well in KGM Gold Complete medium (Lonza, Basel, Switzerland).

After reaching $70 \%$ to $80 \%$ of confluency, cells were pre-incubated in the ab- 
sence (control) or in the presence of increasing concentrations of a cyanobacteria phycocyanin $(0.02 \% ; 0.2 \%$ and $2 \%, v / v)$ for 24 hours. At the end of the pre-incubation period, cells were irradiated or not (control) by UVB at 20 $\mathrm{mJ} / \mathrm{cm}^{2}$ and then incubated for $70 \mathrm{~min}$ in the same experimental conditions.

At the end of the post-irradiation incubation period, mTOR was extracted from cell monolayer and p-mTOR at the position S2448 was then quantified using a specific ELISA assay.

Total proteins contained in the cell lysates were also quantified, by using a spectro-colorimetric method (Bradford method [20]).

Each experimental condition was realized in triplicate $(n=3)$.

\subsection{Telomere Length, Progerin, mTOR Activation and Protein Assays}

\section{Telomere length determination}

For the determination of telomere length, a high-throughput (HT) Q-FISH technique is used according to literature [21] [22]. Briefly, this is a quantitative fluorescence in-situ hybridization method: telomeres are hybridized with fluorescent PNA probe (Alexa488-OO-CCCTAACCCTAACCCTAA, Panagene, Daejeon, Korea) against the telomeric repeats and nuclei are labeled with DAPI (Sigma Aldrich, Saint-Louis, USA). Images were captured by a high-content screen system and the intensity of signal from the PNA probe which is proportional to the length of telomere, is translated to a standard regression curve using a control cell lines.

Cells are seeded in 384-well plates at 15,000 cells/well with 5 replicates of each sample and 8 replicates of each control cell lines then fixed with methanol/acid acetic (3:1). Cells cytoplasm is digested with pepsin treatment and the nuclei are processed for in-situ hybridization with PNA probe and DAPI incubation for DNA staining. After several washings, mounting medium is added and the plate is stored overnight at $4^{\circ} \mathrm{C}$.

Image acquisition ( $\times 40$ lens) and analysis are performed on a high content screening opera system (Perkin Elmer) using the Acapella software. 15 images per well at different positions are captured and results of intensity for each foci are exported to the Columbus 2.4 software (Perkin Elmer). Spot number analyzed per sample was higher than 1000.

Progerin assay

Multiwell plates, especially designed to perform ELISA assays, were incubated overnight at $4^{\circ} \mathrm{C}$ with $100 \mu \mathrm{l}$ of serial dilutions of the standard peptide or of the samples to assay. At the end of this incubation period, non-specific binding sites were saturated by the addition of $300 \mu \mathrm{l}$ per well of a 1\% BSA solution in Phosphate Buffered Solution (PBS, Sigma Aldrich, Saint Louis, USA). After an incubation period of one hour at room temperature, wells were emptied and filled with $100 \mu \mathrm{l}$ of a $1 \%$ BSA solution in PBS containing the anti-human progerin antibody diluted at 1/10,000 (Covalab, Villeurbanne, France). After an incubation period of two hours at room temperature, the wells were washed three times 
with PBS containing $0.1 \%$ of Tween 20 . Wells were then filled with $100 \mu \mathrm{l}$ of a $1 \%$ BSA solution in PBS containing the secondary antibody diluted at 1/2000 (Antibodies-Online, Aachen, Germany) coupled with a peroxidase, and were incubated at room temperature for two hours. At the end of this incubation period, wells were again washed three times with PBS containing $0.1 \%$ of Tween 20 and $100 \mu \mathrm{l}$ of a solution containing a peroxidase substrate were added to each well. After 20 minutes, the peroxidase reaction was terminated by adding $50 \mu \mathrm{l}$ of a $2 \mathrm{~N} \mathrm{H}_{2} \mathrm{SO}_{4}$ solution. The colorimetric signal was analyzed (two wavelengths of reading: 450 and $540 \mathrm{~nm}$ ) by using an appropriate spectrophotometry plate reader (Victor V, Perkin Elmer). For calculations, the signal obtained at $540 \mathrm{~nm}$ was subtracted from those obtained at $450 \mathrm{~nm}$.

mTOR activation assay

mTOR1 complex activation was assessed by measuring phosphorylation of mTOR at the position S2448 using a dedicated ELISA kit (R \& D Systems, Minneapolis, USA) and following manufacturer instructions. Briefly, cell monolayers were scrapped, solubilized in lysis buffer and then allowed to sit on ice for 15 minutes. Crude extracts were then centrifuged at $2000 \times \mathrm{g}$ for 5 minutes. Supernatant were deposited in an ELISA plate previously coated with an antibody that binds mTOR (phosphorylated and unphosphorylated). After a washing away unbound material, a biotinylated detection antibody specific for mTOR phosphorylated at S2448 was used to bind phosphorylated protein. After a washing away unbound material, streptavidin-HRP was added to each well. The complex formed was finally revealed by addition of peroxidase substrate. After incubation, the peroxidase reaction was terminated by adding $50 \mu \mathrm{l}$ of a $2 \mathrm{~N} \mathrm{H}_{2} \mathrm{SO}_{4}$ solution. The colorimetric signal was analyzed (two wavelengths of reading: 450 and $540 \mathrm{~nm}$ ) by using an appropriate spectrophotometry plate reader (Victor V, Perkin Elmer). For calculations, the signal obtained at $540 \mathrm{~nm}$ was subtracted from those obtained at $450 \mathrm{~nm}$.

\section{Protein assay}

Protein assay was performed in cell lysates following the Bradford method [20].

\subsection{Statistics}

Data are expressed as means \pm S.E. of experiments realized at least in triplicate $(\mathrm{n}=3)$, as indicated. The statistical significances were assessed by Student's $\mathrm{t}$ tests or by one way analysis of variance (one way ANOVA) followed by Holm-Sidak's tests (as indicated).

\section{Results and Discussion}

As showed in Figures 1(a)-(c), ultraviolet + blue light (UV + BL) irradiations induced a significant decrease of telomeres' length. Medium and "20th percentile telomeres" displayed a reduced length, i.e. $-18.41 \%(\mathrm{p}<0.01)$ and $-28.15 \%(\mathrm{p}<$ $0.05)$, and the number of "short" telomeres $(<3 \mathrm{Kbp})$ was increased by $+29.4 \%(\mathrm{p}$ $<0.01)$. 


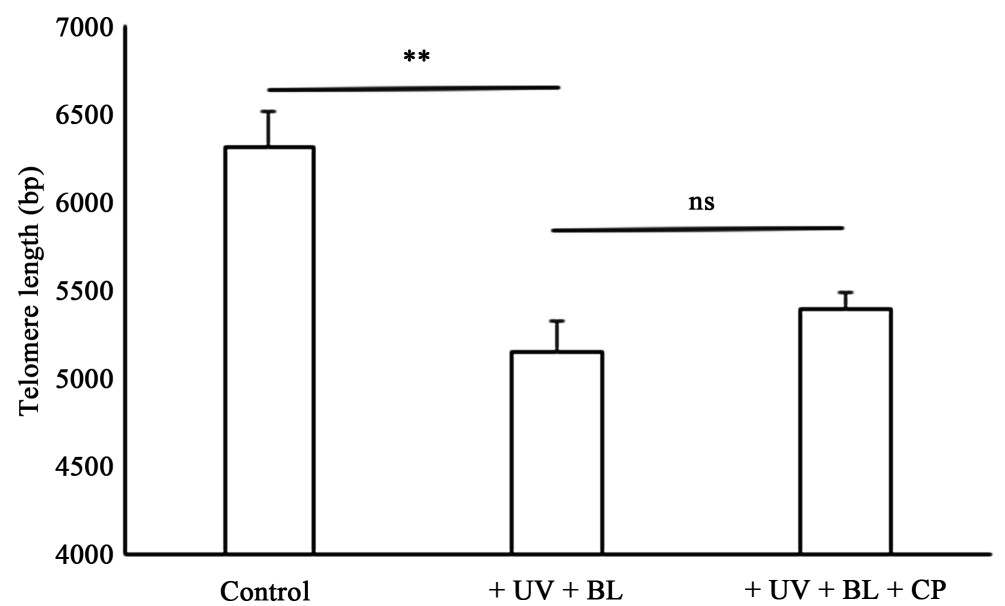

(a)

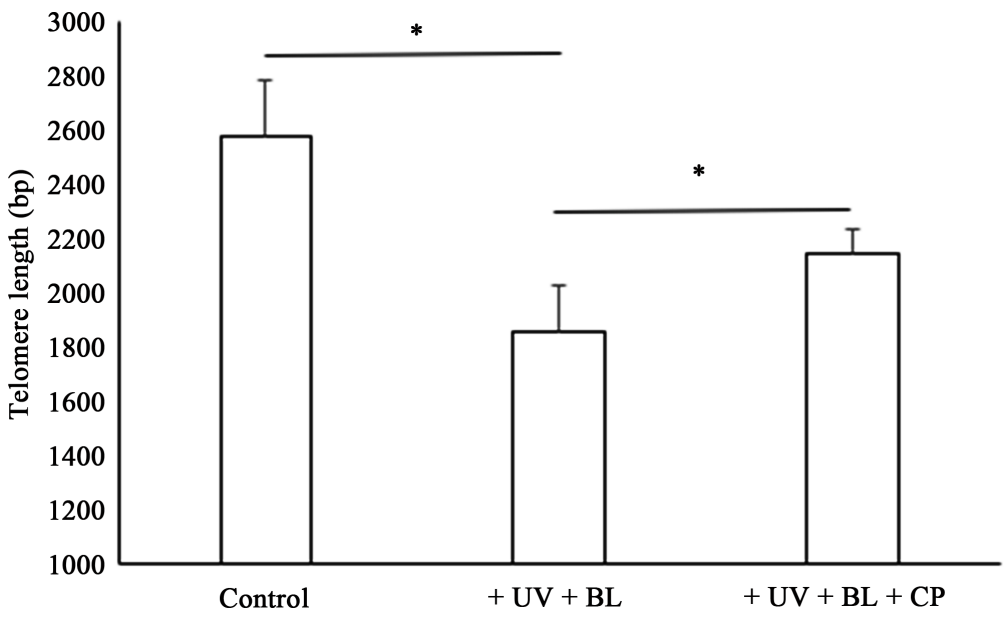

(b)

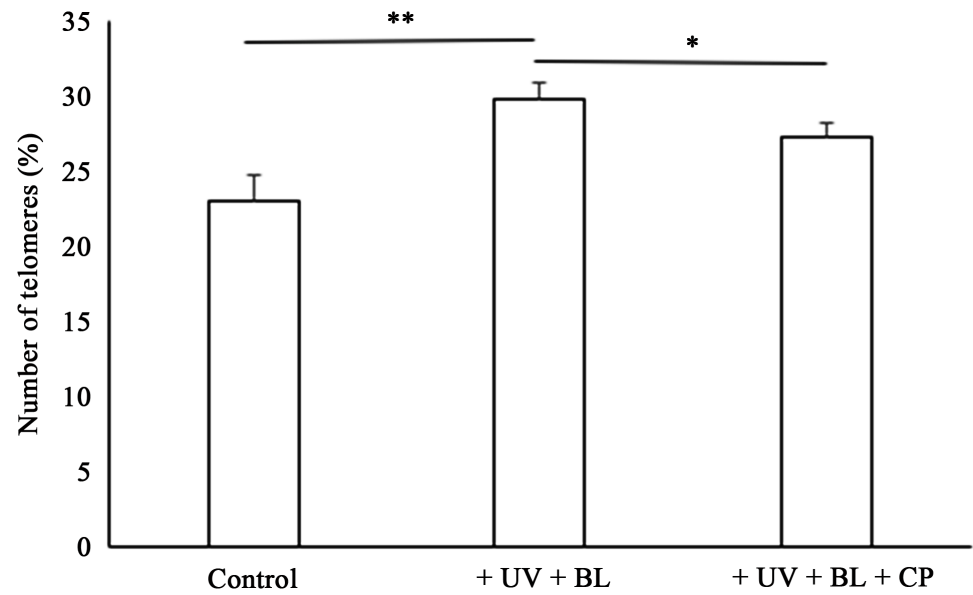

(c)

Figure 1. Impact of UV + Blue Light irradiations on human dermal fibroblasts telomere length; effect of a cyanobacteria phycocyanin. (a) Medium length telomeres; (b) 20th Percentile telomere length; (c) Percentage of short telomeres $(<3 \mathrm{Kbp})$. UV = Ultraviolet irradiation; $\mathrm{BL}=$ Blue Light irradiation; $\mathrm{CP}=$ Cyanobacteria Phycocyanin. ${ }^{*}: \mathrm{p}<0.05$; ${ }^{*}$ : $\mathrm{p}<0.01$; ns: non-significant Data came from one experiment realized in triplicate $(n=3)$. Statistical significance of the observed differences was assessed by Student's t tests. 
Interestingly, we can also note that the tested cyanobacteria phycocyanin was able to counteract the UV + BL effects on the measured parameter by inducing a recovering of $60.48 \%(\mathrm{p}<0.05)$ in telomere length of the "20th percentile telomeres" and by reducing the number of short telomeres by $37.44 \%(\mathrm{p}<0.05)$.

These first results lead to the conclusion that the anti-aging effects of the tested cyanobacteria phycocyanins involve in part the protection of "short" telomeres, and/or their repair. Interestingly our results are in line with the work of Vera and Blasco who showed the association of "short" telomeres with age-related pathologies and cancer as well with reduced lifespan and enhanced mortality [23].

As progerin was also implicated in the aging phenomenon triggering [7], we decided to also study the effect of our cyanobacteria phycocyanin on human dermal fibroblast production.

As showed in Figure 2, UV irradiations significantly increased the fibroblast progerin production by $78.6 \%(\mathrm{p}<0.01)$. In a very interesting way, this result is in line with the work published by Takeuchi and Rünger in 2013 [24] showing by using western blot technics that progerin expression is increased by UVA in young and mature skin fibroblasts. UV irradiations so really seem to act through intracellular pathways involving an increased progeria synthesis to produce their deleterious pro-aging effect on human skin.

As also showed in Figure 2, the cyanobacteria phycocyanin tested at $0.02 \%$; $0.2 \%$ and $2 \%(\mathrm{v} / \mathrm{v})$ was able to significantly counteract the UV-irradiation effects on the fibroblast's progerin production: $-22.3 \%(\mathrm{~ns}), 78.4 \%(\mathrm{p}<0.01)$ and $89.9 \%$ ( $\mathrm{p}<0.01)$, respectively.

This result reinforces the fact that the tested cyanobacteria phycocyanin can provide a really good agent against UV-induced aging, probably through its well-known anti-oxidant properties (oxidative bursts induced in cells by UV no longer need to be demonstrated).

In a very interesting way, results showed in Figure 2 lead us to believe that the anti-aging effect of the tested cyanobacteria phycocyanin could perhaps acts also through other intracellular pathways. In fact, we can observe that the cyanobacteria phycocyanin tested at $0.2 \%(\mathrm{v} / \mathrm{v})$ was also able to reduce the basal fibroblast progerin production: $-28.0 \%(\mathrm{p}<0.05)$. However, several intracellular signaling pathways can be involved in this "basal" effect, for example an action on the abnormal farnesylation of prelamin-A leading to the progerin formation (for a revue, see [23]), and additional works are needed to clarify this issue. We are currently leading some studies aimed to decipher the pathways involved in this effect.

Based on the work of Cao et al. [9] which strongly suggest the implication of the mTOR1 complex in the progerin-induced cellular aging, we then continued our study by analyzing the effect of our cyanobacteria phycocyanin on the UVB-induced mTOR1 activation [25].

As showed in Figure 3, UVB significantly increased the mTOR1 activation rate by $73.2 \%(\mathrm{p}<0.01)$. In the same experimental conditions, the cyanobacteria 


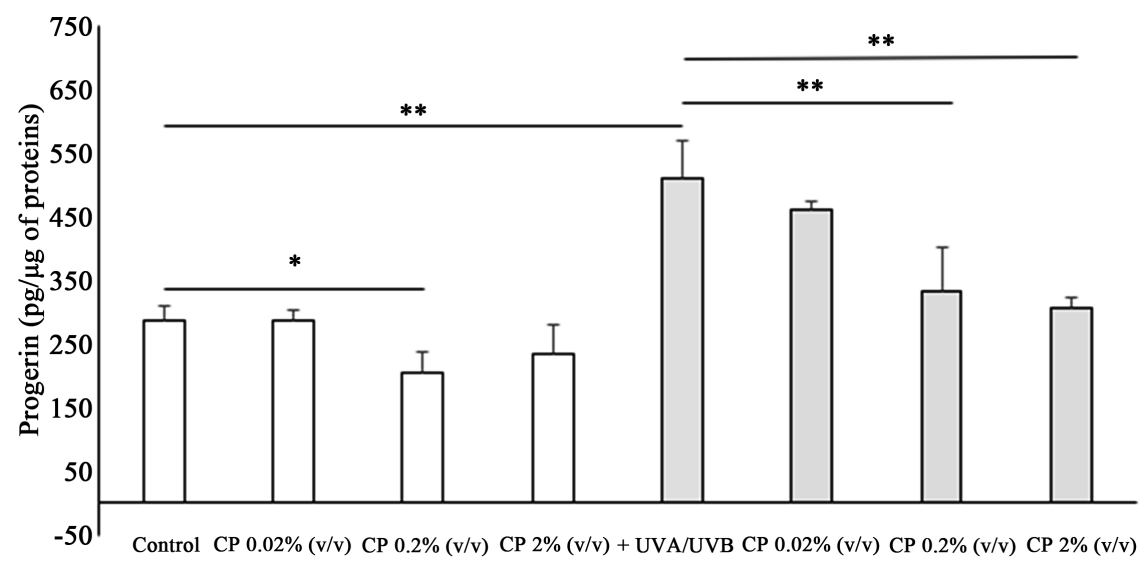

Figure 2. Impact of UVA + UVB irradiations on human dermal fibroblasts progerin production; effect of a cyanobacteria phycocyanin. ${ }^{*}$ : $\mathrm{p}<0.05$; ${ }^{*}$ : $\mathrm{p}<0.01$. Data came from one experiment realized in triplicate $(n=3)$. Statistical significance of the observed differences was assessed by one way analysis of variance (one way ANOVA) followed by Holm-Sidak's tests.

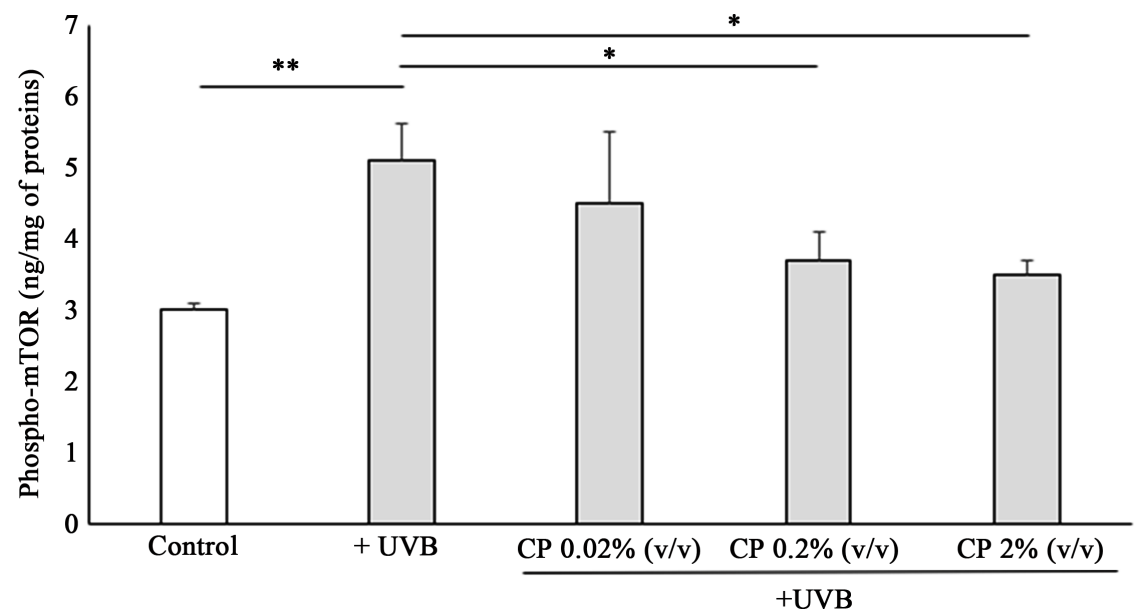

Figure 3. Impact of UVB irradiation on mTOR1 activation rate; effect of a cyanobacteria phycocyanin. ${ }^{*}: \mathrm{p}<0.05 ;{ }^{* *}: \mathrm{p}<0.01$. Data came from one experiment realized in triplicate $(n=3)$. Statistical significance of the observed differences was assessed by one way analysis of variance (one way ANOVA) followed by Holm-Sidak's tests.

phycocyanin tested at $0.02 \% ; 0.2 \%$ and $2 \%(\mathrm{v} / \mathrm{v})$ significantly inhibits the UVB-induced mTOR activation by $30.2 \%(\mathrm{~ns}), 65.4 \%(\mathrm{p}<0.05)$ and $73.8 \%(\mathrm{p}<$ 0.05), respectively.

It is then possible to suggest that the anti-aging potential of our cyanobacteria phycocyanin which was demonstrated at the "telomere" and the "progerin" level could act through the activation rate of the mTOR1 complex, a complex greatly implicated in cell proliferation, growth and differentiation, in mRNA translation and protein synthesis, but also notably in autophagy.

\section{Conclusions}

We showed in this work that a cyanobacteria phycocyanin was able, in different 
models of human normal skin cells, to counteract 1) the telomeres' length reduction induced by $\mathrm{UV}+\mathrm{BL}$ irradiations, 2) the progerin production induced by UV irradiation but not only, because it also displayed an inhibitory effect on the "basal" progerin production, and 3) the effect of UVB on the activation rate of the mTOR1 complex, a key regulator of the cellular physiopathology. Additional studies are currently running in our laboratories in order to better understand the anti-aging and photo-protective effect of this cyanobacteria phycocyanin on skin cells.

\section{Acknowledgements}

Special thanks to the company "Life Length" (Madrid, Spain) for their helpful technical assistance.

\section{References}

[1] Pages, F., Guedes, A., Amaro, H., Kijjoa, A. and Vasconcelos, V. (2019) Phycobiliproteins from Cyanobacteria: Chemistry and Biotechnological Applications. Biotechnology Advances, 37, 422-443. https://doi.org/10.1016/j.biotechadv.2019.02.010

[2] Chang, A. (2016) Expanding Our Understanding of Human Skin Aging. Journal of Investigative Dermatology, 136, 897-899. https://doi.org/10.1016/j.jid.2016.02.020

[3] Kmiecik, B., Skotny, A., Batycka, M., Wawrzaszek, R. and Rybak, Z. (2013) Influence of Oxidative Stress on Tissue Regeneration. Polymers in Medicine, 43, 191-197.

[4] Irshad, M. and Chaudhuri, P. (2002) Oxidant-Antioxidant System: Role and Significance in Human. Indian Journal of Experimental Biology, 40, 1233-1239.

[5] Gunes, S., Tamburaci, S., Dalay, M. and Deliloglu Gurhan, I. (2017) In Vitro Evaluation of Spirulina platensis Extract Incorporated Skin Cream with Its Wound Healing and Antioxidant Activities. Pharmaceutical Biology, 55, 1824-1832. https://doi.org/10.1080/13880209.2017.1331249

[6] Wu, L., Lin, Y., Yang, S., Weng, Y. and Tsai, Y. (2011) Antimelanogenic Effect of c-Phycocyanins through Modulation of Tyrosinase Expression by Upregulation of ERK and Downregulation of p38 MAPK Signaling Pathways. Journal of Biomedical Science, 18, 1-9. https://doi.org/10.1186/1423-0127-18-74

[7] Cao, K., Blair, C., Faddah, C., Kieckhaefer, J., Olive, M., Erdos, M., Nabel, E. and Collins, F. (2011) Progerin and Telomere Dysfunction Collaborate to Trigger Cellular Senescence in Normal Human Fibroblasts. Journal of Clinical Investigation, 121, 2833-2844. https://doi.org/10.1172/JCI43578

[8] Sondenheimer, K. and Krutmann, J. (2018) Novel Means for Photoprotection. Frontiers of Medicine, 5, 162. https://doi.org/10.3389/fmed.2018.00162

[9] Cao, K., Graziotto, J., Blair, C., Mazzulli, J., Erdos, M., Krainc, D. and Collins, F. (2011) Rapamycin Reverses Cellular Phenotypes and Enhances Mutant Protein Clearancein Hutchinson-Gilford Progeria Syndrome Cells. Science Translational Medicine, 3, 89ra58. https://doi.org/10.1126/scitranslmed.3002346

[10] Benson, E., Lee, S. and Aaronson, S. (2010) Role of Progerin-Induced Telomere Dysfunction in HGPS Premature Cellular Senescence. Journal of Cell Science, 123, 2605-2612. https://doi.org/10.1242/jcs.067306

[11] Kudlow, B., Stanfel, M., Burtner, C., Johnston, E. and Kennedy, B. (2008) Suppression of Proliferative Defects Associated with Processing-Defective Lamin A Mutants 
by hTERT or Inactivation of p53. Molecular Biology of the Cell, 19, 5238-5248. https://doi.org/10.1091/mbc.e08-05-0492

[12] Varela, I., Cadinanos, J., Pendas, A., Gutierez-Fernandez, A., Folgueras, A., Sanchez, L., Zhou, Z., Rodriguez, F., Stewart, C., Vega, J., Tryggvason, K., Freije, J. and Lopez-Otin, C. (2005) Accelerated Ageing in Mice Deficient in Zmpste24 Protease Is Linked to p53 Signalling Activation. Nature, 437, 564-568.

https://doi.org/10.1038/nature04019

[13] Musich, P. and Zou, Y. (2009) Genomic Instability and DNA Damage Responses in Progeria Arising from Defective Maturation of Prelamin A. Aging, 1, 28-37. https://doi.org/10.18632/aging.100012

[14] Gonzalez-Suarez, I., Redwood, A. and Gonzalo, S. (2009) Loss of A-Type Lamins and Genomic Instability. Cell Cycle, 8, 3860-3865.

https://doi.org/10.4161/cc.8.23.10092

[15] Jung, C., Ro, S., Cao, J., Otto, N. and Kim, D. (2010) MTOR Regulation of Autophagy. FEBS Letters, 584, 1287-1295. https://doi.org/10.1016/j.febslet.2010.01.017

[16] Laplante, M. and Sabatini, D. (2009) An Emerging Role of mTOR in Lipid Biosynthesis. Current Biology, 19, R1046-R1052. https://doi.org/10.1016/j.cub.2009.09.058

[17] Rosner, M. and Hengstschläger, M. (2011) mTOR Protein Localization Is Cell Cycle Regulated. Cell Cycle, 10, 3608-3610. https://doi.org/10.4161/cc.10.20.17855

[18] Laplante, M. and Sabatini, D. (2012) MTOR Signaling in Growth Control and Disease. Cell, 149, 274-293. https://doi.org/10.1016/j.cell.2012.03.017

[19] Shimobayashi, M. and Hall, M. (2014) Making New Contacts: The mTOR Network in Metabolism and Signalling Crosstalk. Nature Reviews Molecular Cell Biology, 15, 155-162. https://doi.org/10.1038/nrm3757

[20] Bradford, M. (1976) A Rapid and Sensitive Method for the Quantitation of Microgram Quantities of Protein Utilizing the Principle of Protein-Dye Binding. Analytical Biochemistry, 7, 248-254. https://doi.org/10.1006/abio.1976.9999

[21] Canela, A., Vera, E., Klatt, P. and Blasco, M. (2007) High-Throughput Telomere Length Quantification by FISH and Its Application to Human Population Studies. Proceedings of the National Academy of Sciences of the United States of America, 104, 5300-5305. https://doi.org/10.1073/pnas.0609367104

[22] Kimura, A., Stone, R., Hunt, S., Skurnick, J., Lu, X., Cao, X. and Harley, C. (2010) Measurement of Telomere Length by the Southern Blot Analysis of Terminal Restriction Fragment Lengths. Nature Protocols, 5, 1596-1607. https://doi.org/10.1038/nprot.2010.124

[23] Vera, E. and Blasco, M. (2012) Beyond Average: Potential for Measurement of Short Telomeres. Aging, 4, 379-391. https://doi.org/10.18632/aging.100462

[24] Young, S., Meta, M., Yang, S. and Fong, L. (2006) Prelamin A Farnesylation and Progeroid Syndromes. The Journal of Biological Chemistry, 281, 39741-39745. https://doi.org/10.1074/jbc.R600033200

[25] Lin, F., Xu, W., Guan, C., Zhou, M., Hong, W., Fu, L., Liu, D. and Xu, A. (2012) Niacin Protects against UVB Radiation-Induced Apoptosis in Cultured Human Skin Keratinocytes. International Journal of Molecular Medicine, 29, 593-600. https://doi.org/10.3892/ijmm.2012.886 